\title{
Laparoscopy significantly improves the perceived preoperative stage of gastric cancer
}

\author{
Guy R.J.C. Blackshaw ${ }^{1}$, Jonathan D. Barry ${ }^{1}$, Paul Edwards ${ }^{1}$, Miles C. Allison ${ }^{2}$, Gerald V. Thomas ${ }^{3}$, \\ and WYN G. LEWIS ${ }^{1}$ \\ ${ }^{1}$ Department of Surgery, Royal Gwent Hospital, Cardiff Road, Newport, NP20 2UB, UK \\ ${ }^{2}$ Department of Gastroenterology, Royal Gwent Hospital, Newport, UK \\ ${ }^{3}$ Department of Radiology, Royal Gwent Hospital, Newport, UK
}

\begin{abstract}
Background. The aim of this study was to examine the accuracy of laparoscopy in staging patients with gastric cancer in comparison with preoperative computed tomography (CT) examination.

Methods. One hundred patients out of a consecutive series of 258 patients with gastric adenocarcinoma underwent a preoperative staging CT followed by a staging laparoscopy. The strengths of the agreement between the CT stage, the laparoscopic stage, and the final histopathological stage were determined by the weighted Kappa statistic (Kw).

Results. The strengths of agreement between the CT stage and the final histopathological stage were $\mathrm{Kw}=0.336(95 \%$ confidence interval $[\mathrm{CI}] ; 0.172-0.5 ; P=0.0001)$ for $\mathrm{T}$ stage and $0.378(95 \% C I ; 0.226-0.53 ; P=0.0001)$ for $M$ stage, compared with $0.455(95 \% \mathrm{CI} ; 0.301-0.609 ; P=0.0001)$ and 0.73 (95\% CI; $0.596-0.864 ; P=0.0001)$ for the laparoscopic $T$ and $M$ stages, respectively. Unsuspected metastases that were not detected by CT, were found in 21 patients at laparoscopy, all of whom had $\mathrm{T} 3$ or $\mathrm{T} 4$ locally advanced tumors evident on CT.

Conclusion. Preoperative laparoscopic staging of gastric cancer is indicated for potential surgical candidates with locally advanced disease in the absence of metastases on CT.
\end{abstract}

Key words Laparoscopy $\cdot$ Staging $\cdot$ Gastric cancer

\section{Introduction}

Gastric cancer is now the second commonest cancer worldwide, accounting for 11000 deaths per annum in the United Kingdom alone [1]. The stage of disease at presentation is the most important prognostic factor.

Offprint requests to: W.G. Lewis An original article presented at The British Society of Gastroenterology, Birmingham, 2002 (Gut 2002; 50 [Suppl. II] A8). Received: April 22, 2003 / Accepted: August 6, 2003
Despite the introduction of open access gastroscopy, as many as one in three British patients continue to present with advanced disease that is incurable by means of surgery $[2,3]$. Thorough and accurate staging of patients is of paramount importance in the selection of patients for whom surgery could be potentially curative [4].

The recent large regional audit of esophagogastric cancer in Wales revealed that many surgeons continue to undertake small caseloads; staging laparoscopy was performed in $16 \%$ of cases; and no fewer than $23 \%$ of patients underwent open-and-close operations [3]. Staging protocols for patients with gastric cancer vary, not only from hospital to hospital within the United Kingdom, but from east to west worldwide. While computed tomography (CT) remains the most widely used imaging technique for the preoperative staging of patients with gastric cancer, this modality is limited in its ability to detect local invasion and lymph node metastases [5-8]. Moreover, we have already shown that specialized radiological support improves the perceived preoperative stage of gastric cancer and reduces the rate of open-and-shut laparotomy [9].

Although routine laparoscopy is now advocated in the preoperative staging of gastric cancer [4], there are discrepant results in the literature as to the clinical value of this procedure [10]. The aims of this study therefore, were to compare the perceived preoperative stage of gastric cancer as determined by CT and laparoscopy, and to compare this perceived stage with the final true histopathological stage. It was our hypothesis that laparoscopy would improve the accuracy of our preoperative staging process.

\section{Patients and methods}

Between October 1, 1995, and April 30, 2000, we studied 100 patients (out of a consecutive series of 258 
patients) who underwent both preoperative spiral CT and operative assessment. Of the remaining 158 patients, 102 had either clinical or radiological evidence of metastatic disease or were unfit for surgery. Fifty-six patients underwent surgery without preoperative staging laparoscopy, as CT had shown T1 or T2, N0M0 disease. Each patient had a preoperative histological diagnosis of gastric adenocarcinoma established by gastroscopy and biopsy. Of the 100 patients undergoing laparoscopy, the median age was 70 years (range, 35 to 86 years). Fifty-nine were male and 41 were female. The CT examinations were performed on a Siemens Somatom plus four CT system (Munich, Germany). All patients underwent a contrast-enhanced spiral CT within 3 weeks of operation as per the following protocol: 30 min before CT, after an overnight fast, patients were asked to drink $250 \mathrm{ml}$ of Microcat contrast medium (Guernet, 95943 Roissy DdCr Cedex, France) to opacify the small bowel. All patients were then asked to take as much of a 1000-ml water load as they could tolerate. One hundred $\mathrm{ml}$ of intravenous sodium iothalamate (Niopam 300; Merck Pharmaceuticals, Middlesex, UK) was delivered by power injector, at a rate of $3 \mathrm{ml}$ per $\mathrm{s}$, through a cannula in the antecubital fossa. All patients were examined in the supine position. Initially, a volume acquisition of the thorax was performed, using 10-mm slice thicknesses, a reconstruction index of $8 \mathrm{~mm}$, and a table speed of $10 \mathrm{~mm}$ per s. A second volume acquisition of the abdomen, from the dome of the diaphragm to the pelvic brim, was performed, using 10-mm slice thicknesses with a reconstruction index of $5 \mathrm{~mm}$. This meant that slices were acquired every $10 \mathrm{~mm}$, but reconstructed at $5-\mathrm{mm}$ intervals, which produced overlapping slices. The second volume was timed to begin liver imaging $60-70$ s after the onset of the injection of contrast medium.

Patients were allocated to the next available consultant radiologist's CT list. For the purposes of this study, adjacent organs were considered to be involved if there was CT evidence of direct spread of the tumor into adjacent organs. Specific areas of fat plane effacement were also compared with adjacent evidence of fat plane preservation. Lymph nodes were considered to be involved if they were greater than $1 \mathrm{~cm}$ in diameter [11-13]. A radiological audit meeting was held prior to the start of this study in order to review the accuracy of $\mathrm{CT}$ as a staging investigation for patients with gastric cancer at the Royal Gwent Hospital. At this time, all of the consultant radiologists were informed of the TNM pathological staging classification $[14,15]$ and asked to construct their reports in this structured fashion.

One hundred patients who were considered on CT criteria to have advanced stage disease (T3 or T4, N1 or N2, and equivocal M1) underwent a preoperative staging laparoscopy. No patients received preoperative neoadjuvant chemotherapy. Forty-six of these patients subsequently underwent a potentially curative radical D2 gastrectomy, 5 underwent a palliative resection, and the remaining 49 patients underwent either laparoscopy alone with biopsy $(n=34)$, palliative bypasses $(n=7)$, or laparotomy only $(n=8)$. All patients underwent operative assessment, as suggested by Rohde et al. [16]. In the course of 11 operations, en-bloc splenectomy $(n=5)$, distal pancreatectomy $(n=3)$, and transverse colectomy $(n=7)$ were performed.

\section{Technique of laparoscopy}

Laparoscopy was performed with the patient under general anesthetic. The patient was positioned as for an open upper abdominal procedure, and the operating table was repositioned according to the intraabdominal region to be examined. A $1-\mathrm{cm}$ subumbilical incision was made and carbon dioxide insufflation was performed through a Veress needle to an insufflation pressure of $12 \mathrm{mmHg}$. A $10-\mathrm{mm}$ disposable cannula was inserted and laparoscopy was performed using a $0^{\circ}$ telescope. A supplementary disposable 5-mm cannula was inserted in the patient's left upper quadrant to allow the use of grasping and biopsy forceps. A thorough inspection of all four quadrants of the peritoneal cavity was performed, and biopsies were taken from any tissue suspected to be tumor. The lesser sac was not inspected routinely. No patients underwent laparoscopic ultrasound examination.

\section{Statistical analysis}

The findings from CT and staging laparoscopy were compared with the final stage. The final stage was based on the combination of intraoperative surgical assessment and postoperative histopathological stage. Agreement between the perceived preoperative stage of gastric cancer as determined by CT, laparoscopy, and the final stage was determined using the weighted Kappa statistic (Kw) [17]. The value of Kappa has a maximum of 1.00 when agreement is perfect, a value of zero indicates no agreement better than chance, and negative values show worse than chance agreement. We tested the hypothesis $\mathrm{Kw}=0$ and assessed the value of $\mathrm{Kw}$ for strength of agreement according to the guidelines of Landis and Koch (a value between zero and 0.2 is defined as being poor agreement, between 0.2 and 0.4 is termed fair agreement, 0.4 to 0.6 moderate, 0.6 to 0.8 good, and 0.8 to 1 is very good agreement) [18]. The sensitivity and specificity, positive predictive value (PPV), and negative predictive value (NPV) were also calculated. Categorical data were further analyzed by means of the $\chi^{2}$ distribution [17]. Data analysis was car- 
ried out with the Statistical Package for Social Sciences (SPSS) version 11 (SPSS, Chicago, IL, USA).

\section{Results}

\section{Accuracy of staging of tumor infiltration (T stage)}

(Tables 1 and 2)

By statistical analysis, the strengths of agreement between the perceived $\mathrm{T}$ stage on $\mathrm{CT}$ and the final histopathological stage were fair $(\mathrm{Kw}=0.336 ; 95 \%$ confidence interval [CI], 0.172 to $0.50 ; P=0.0001$ ) for CT, compared with moderate $(\mathrm{Kw}=0.455 ; 95 \% \mathrm{CI}$, 0.301 to $0.609 ; P=0.0001$ ) for laparoscopy.

\section{Accuracy of staging of distant metastases (M stage) (Tables 2 and 3)}

The strengths of agreement between the perceived $M$ stage and the final histopathological stage were fair $(\mathrm{Kw}=0.378 ; 95 \% \mathrm{CI}, 0.226$ to $0.53 ; P=0.0001)$ for $\mathrm{CT}$,

Table 1. Accuracy of computed tomographic and laparoscopic staging of tumor infiltration ( $\mathrm{T}$ stage)

Final histopathological/operative stage

\begin{tabular}{llccc}
\hline Stage by CT & & T1/2 & T3 & T4 \\
& T1/2 & $7^{\mathrm{a}}$ & 2 & 1 \\
& T3 & 4 & $24^{\mathrm{a}}$ & 17 \\
& T4 & 4 & 12 & $29^{\mathrm{a}}$
\end{tabular}

Final histopathological/operative stage

\begin{tabular}{llccc}
\hline Stage by & & $\mathrm{T} 1 / 2$ & $\mathrm{~T} 3$ & $\mathrm{~T} 4$ \\
laparoscopy & $\mathrm{T} 1 / 2$ & $8^{\mathrm{a}}$ & 2 & 0 \\
& $\mathrm{~T} 3$ & 8 & $33^{\mathrm{a}}$ & 4 \\
& $\mathrm{~T} 4$ & 0 & 19 & $26^{\mathrm{a}}$
\end{tabular}

Figures are numbers of patients

${ }^{a}$ Correct staging compared with good $(\mathrm{Kw}=0.73 ; 95 \% \mathrm{CI}, 0.596$ to $0.864 ; P=0.0001)$ for laparoscopy. The strengths of agreement between the perceived $M$ stage with respect to liver metastases were good $(\mathrm{Kw}=0.704 ; 95 \% \mathrm{CI}$, $0.482-0.926 ; P=0.0001)$ for CT, compared with very $\operatorname{good}(\mathrm{Kw}=0.813 ; 95 \% \mathrm{CI}, 0.635-0.991 ; P=0.0001)$ for laparoscopy. The strengths of agreement between the perceived M stage with respect to peritoneal metastases were fair $(\mathrm{Kw}=0.375 ; 95 \% \mathrm{CI}, 0.199-0.551 ; P=$ $0.0001)$ for $\mathrm{CT}$, compared with $\operatorname{good}(\mathrm{Kw}=0.724$; 95\% CI, 0.574-0.874; $P=0.0001$ ) for laparoscopy. Twenty patients were found to have distant metastases on laparoscopy that were undetected by CT (15 peritoneum alone, 2 liver alone, 3 liver and peritoneum). One patient was found to have posterior invasion precluding resection. Of the 19 patients with missed peritoneal disease or local invasion, 4 patients were staged as T3/ 4N0M0 and 15 patients were staged as T3/4N1/2M0. Of the 5 patients with missed liver metastases on CT, 1 patient was staged as T4N0M0, and the other 4 patients were staged as $\mathrm{T} 3 / 4 \mathrm{~N} 1 / 2 \mathrm{M} 0$.

Table 3. Accuracy of computed tomographic and laparoscopic staging of distant metastases (M stage)

Final histopathological/operative stage

\begin{tabular}{llccc}
\hline Stage by CT & M0 & M1P & M1L \\
& M0 & $57^{\mathrm{a}}$ & 23 & 6 \\
& M1P & 0 & $11^{\mathrm{a}}$ & \\
M1L & 0 & & $9 \mathrm{a}$
\end{tabular}

Final histopathological/operative stage

\begin{tabular}{llccc}
\hline Stage by & & M0 & M1P & M1L \\
laparoscopy & M0 & $57^{\mathrm{a}}$ & 11 & 3 \\
& M1P & 0 & $1^{\mathrm{a}}$ & \\
& M1L & 0 & & $11^{\mathrm{a}}$
\end{tabular}

Figures are numbers of patients

M0, no distant metastases; M1P, peritoneal metastases; M1L, liver metastases

${ }^{a}$ Correct staging

Table 2. Sensitivity, specificity, predictive values, and accuracy of computed tomographic and laparoscopic staging

\begin{tabular}{|c|c|c|c|c|c|c|c|c|c|c|}
\hline & \multicolumn{5}{|c|}{ Computed tomography } & \multicolumn{5}{|c|}{ Laparoscopy } \\
\hline & Sens. & Spec. & PPV & NPV & Acc. & Sens. & Spec. & PPV & NPV & Acc. \\
\hline $\mathrm{T} 1 / 2$ & 70 & 91 & 47 & 96 & 89 & 80 & 91 & 50 & 98 & 90 \\
\hline $\mathrm{T} 3$ & 53 & 75 & 63 & 66 & 65 & 73 & 62 & 61 & 74 & 67 \\
\hline $\mathrm{T} 4$ & 64 & 67 & 62 & 70 & 66 & 58 & 93 & 87 & 73 & 77 \\
\hline M1 & 84 & 64 & 36 & 95 & 68 & 94 & 80 & 71 & 96 & 85 \\
\hline M1P & 73 & 71 & 32 & 93 & 70 & 88 & 83 & 68 & 95 & 85 \\
\hline M1L & 69 & 90 & 60 & 93 & 86 & 79 & 93 & 73 & 95 & 90 \\
\hline
\end{tabular}

Figures are percentages

Sens., sensitivity; Spec., specificity; PPV, positive predictive value; NPV, negative predictive value;

Acc., accuracy; M1P, peritoneal metastases; M1L, liver metastases 


\section{Outcome after false-negative laparoscopy}

Eight patients who had undergone laparoscopy underwent open-and-close laparotomy due to unsuspected inoperable disease. Unsuspected posterior local tumor invasion was found in 5 patients, while undetected liver metastases were discovered in 3 patients at laparotomy. None of the 100 patients who underwent laparoscopy suffered any significant postoperative morbidity or mortality.

\section{Outcome in patients undergoing surgery without preoperative staging laparoscopy}

Of the 56 patients who underwent surgery without having a preoperative staging laparoscopy, 49 underwent operative resection (38 R0 D2 resections), 1 patient underwent a planned bypass, and 6 patients underwent open-and-close laparotomies. All 6 patients had locally advanced T4 disease on CT, 2 of them being staged T4N0M0, and the other 4 patients were staged as T4N2M0.

\section{Discussion}

Routine staging laparoscopy is now advocated following CT and endoluminal ultrasound prior to consideration of radical surgery in patients with gastric cancer $[4,19$ 21]. Nevertheless, the role of laparoscopy as a routine staging strategy remains controversial, and none of the above reports have assessed the true statistical accuracy of laparoscopy when compared with CT and the final histopathological stages of patients' tumors. The principal findings of this study were that both CT and laparoscopy agreed significantly with histopathological stages. Laparoscopy held a modest $(20 \%)$ advantage over CT in assessing $\mathrm{T}$ stage, and a statistically significant twofold advantage over $\mathrm{CT}$ in assessing the presence of peritoneal metastases. The corresponding clinical benefit was that 21 patients were found to have distant metastases on laparoscopy that were undetected on CT, and were saved unnecessary laparotomies.

The most important goal of preoperative staging investigations is the detection of distant metastases. Liver metastases are reported to be relatively uncommon at presentation in patients with gastric cancer $(8 \%-10 \%)$, although as many as one in two metastases are missed on CT alone [10,22]. Furthermore, interobserver variation exists, even among experienced radiologists, in the interpretation of subtle radiological signs on CT $[9,10]$. The ideal noninvasive staging protocol for patients with gastric cancer is obtained with a combination of helical CT and endogastric ultrasound examination. As far as we are aware, however, there are no reports of the added benefit of laparoscopy in this context.
The striking feature of the major published reports regarding the value of laparoscopy in staging patients with gastric cancer is the extreme variability of the results. Most of the data have emerged from centers with a high incidence of advanced gastric cancer. In Japan, where more than $60 \%$ of the gastric cancers diagnosed are early tumors, staging laparoscopy remains very rare. By contrast, advanced disease precluding curative surgical resection undetected by preoperative CT has been described in $2 \%-37 \%$ of patients in the Western world [23-37]. The reported overall accuracy of the perceived preoperative stage of gastric cancer, as determined by laparoscopy allied to CT, ranges from $83 \%$ to $98 \%$, with alterations in management described in $6 \%-42 \%$ of patients. A metaanalysis of the 17 major reported series, examining 2512 patients, reveals a significant correlation between the accuracy of staging CT and the diagnostic yield of laparoscopy $(r=-0.573$; $P=0.041)$.

More recently, laparoscopic ultrasound examination in addition to CT and laparoscopy has been championed by some authors, as a further benefical staging modality $[38,39]$. Previously undetected liver metastases have been detected in as many as $8 \%$ of patients [38], although the literature does not support this view universally [40]. In our experience, the overall accuracy of the perceived preoperative stage of gastric cancer as determined by the combination of laparoscopy and CT was $92 \%$, with alterations in management occurring in $21 \%$ of patients. Three patients in this series were found to have liver metastases that were missed by both CT and laparoscopy. Arguably, these lesions may have been detected by laparoscopic ultrasound, which would therefore have influenced management in a further $3 \%$ of patients.

In conclusion, optimal preoperative staging of gastric cancer, using helical CT followed by laparoscopy where appropriate, selects patients for whom radical surgery can be potentially curative. The true added value of laparoscopy can only be unequivocally proven by a prospective randomized controlled trial in patients with tumors presumed to be of stage III and IV on CT criteria, which, if the findings of this study are representative, may be difficult to justify on ethical grounds. However, it is apparent that the judicious use of laparoscopy can improve the perceived preoperative stage of gastric cancer two-fold when compared with CT alone, and, until the sensitivity and specificity of radiological imaging modalities approach 100\%, laparoscopy will continue to provide upper gastrointestinal surgeons with important additional staging information.

Acknowledgments We thank Dr. Barry Nix, B.Sc., Ph.D., C.Stat., of the Department of Epidemiology, Statistics, and Public Health, University of Wales College 
of Medicine, Heath Park, Cardiff, for his advice on the statistical aspects of this study.

\section{References}

1. Coleman MP, Esteve J, Damiecki P, Arslan A, Renard H. Trends in cancer incidence and mortality. IARC Sci Publ 1993;12:193228.

2. Allum WH, Powell DJ, McConkey CC, Fielding JWL. Gastric cancer: a 25-year review. Br J Surg 1989;76:535-40.

3. Pye JK, Crumplin MKH, Foster ME, Biffin A, Charles J. Oneyear survey of carcinoma of the oesophagus and stomach in Wales. Br J Surg 2001;88:278-85.

4. Allum WH, Griffin SM, Watson A, Colin-Jones D. Guidelines for the management of oesophageal and gastric cancer. Gut 2002; 50(Suppl V):v1-v23.

5. Fraser I, Nash R, James DC. Computed tomography in gastric cancer. Br J Surg 1985;72:249-50.

6. Cook AO, Levine BA, Sirinek KR, Gaskill HV. Evaluation of gastric adenocarcinoma: abdominal computed tomography does not replace celiotomy. Arch Surg 1986;121:603-6.

7. Sussman SK, Halvorsen RA, Illescas FF, Cohan RH, Saeed M, Silverman WM, et al. Gastric adenocarcinoma: CT versus surgical staging. Radiology 1988;167:335-40.

8. Andaaker L, Morales O, Hojer H, Backstrand B, Borch K, Larsson J. Evaluation of pre-operative computed tomography in gastric malignancy. Surgery 1991;109:132-5.

9. Barry JD, Edwards P, Lewis WG, Dhariwal D, Thomas GV. Special interest radiology improves the perceived preoperative stage of gastric cancer. Clin Radiol 2002;57:984-8.

10. Martin IG. Staging of esophageal and gastric carcinoma. In: Daly JM, Hennessy TPJ, Reynolds JV, editors. Management of upper gastrointestinal Cancer. London: W.B. Saunders; 1999.

11. Vallgren S, Hedenbro J, Gotberg S, Walther B. Preoperative computed tomography for evaluation of tumour growth in patients with gastric cancer. Acta Chir Scand 1985;151:571-3.

12. Botet J, Lightdale CJ, Zauber AG, Gerdes H, Winawer SJ, Urmacher C, et al. Preoperative staging of gastric cancer: comparison of endoscopic US and dynamic CT. Radiology 1991;181: 426-32.

13. Maruyama M, Baba M. Gastric carcinoma: radiology of the upper gastrointestinal tract. Radiol Clin North Am 1994;32:1233-52.

14. Kennedy BJ. The unified international gastric cancer staging classification. Scand J Gastroenterol 1987;22(Suppl 133):11-3.

15. Sobin LH, Wittekind Ch, editors. UICC TNM Classification of malignant tumours. Fifth Ed. New York: John Wiley and Sons; 1997.

16. Rohde H, Rau E, Koster R, Gebbensleben B, Stutzer H. A surgeon's checklist for a standardized staging procedure in patients with cancer of the stomach. Scand J Gastroenterol 1987; 22(Suppl 133):36-9.

17. Altman DG. Practical statistics for medical research. London: Chapman \& Hall/CRC; 1991

18. Landis JR, Koch GG. The measurement of observer agreement for categorical data. Biometrics 1977;33:159-74.

19. Stell DA, Carter CR, Stewart I, Anderson JR. Prospective comparison of laparoscopy, ultrasonography and computed tomography in the staging of gastric cancer. Br J Surg 1996;83:1260-2.

20. Molloy RG, McCourtney JS, Anderson JR. Laparoscopy in the management of patients with cancer of the gastric cardia and oesophagus. Br J Surg 1995;82:352-4.

21. Hunerbein M, Rau B, Schalg PM. Laparoscopy and laparoscopic ultrasound for staging of upper gastrointestinal tumours. Eur J Surg Oncol 1995;21:50-5.
22. Watt I, Stewart I, Anderson E, Bell G, Anderson JR. Laparoscopy, ultrasound and computed tomography in cancer of the oesophagus and gastric cardia: a prospective comparison for detecting intra-abdominal metastases. Br J Surg 1989;76:1036-40.

23. Asencio F, Aguilo J, Salvador JL, Villar A, De la Morena E, Ahamad M, et al. Video-laparoscopic staging of gastric cancer. A prospective multicenter comparison with noninvasive techniques. Surg Endosc 1997;11:1153-8.

24. Bemelman WA, van Delden OM, van Lanschot TT, de Wit LT, Smits NJ, Fockens P, et al. Laparoscopy and laparoscopic ultrasonography in staging carcinoma of the oesophagus and gastric cardia. J Am Coll Surg 1995;181:421-5.

25. Bonavina L, Incarbone R, Lattuda E, Segalin A, Cesana B, Peracchia A. Preoperative laparoscopy in management of patients with carcinoma of the oesophagus and of the oesophagogastric junction. J Surg Oncol 1997;65:171-4.

26. Burke EC, Karpeth MS, Conlon KC, Brennan MF. Laparoscopy in the management of gastric adenocarcinoma. Ann Surg 1997; 225:262-7.

27. Charukhchyan SA, Lucas GW. Laparoscopy and lesser sac endoscopy in gastric carcinoma operability assessment. Am Surg 1998; 64:160-4.

28. D’Ugo DM, Persani R, Caracciolo F, Roconi P, Coco C, Picciocchi A. Selection of locally advanced gastric carcinoma by preoperative staging laparoscopy. Surg Endosc 1997;11:1159-62.

29. Dagnini G, Caldironi MW, Marin G, Buzzaccarinin O, Tremolada C, Ruol A. Laparoscopy in abdominal staging of oesophageal carcinoma. Report of 369 cases. Gastrointest Endosc 1986;32:4002.

30. Kriplani AK, Kapur BM. Laparoscopy for preoperative staging and assessment of operability in gastric carcinoma. Gastrointest Endosc 1991:37:441-3.

31. Lowy AM, Mansfield PF, Leach SD, Ajani J. Laparoscopic staging for gastric cancer. Surgery 1996;119:611-4.

33. O'Brien MG, Fitzgerald EF, Lee G, Crowley M, Shanahan F, O'Sullivan GC. A prospective comparison of laparoscopy and imaging in the staging of esophagogastric cancer before surgery. Am J Gastroenterol 1995;90:2191-4.

34. Possik RA, Franco RA, Pires DR, Wohnrath DR, Ferreira EB. Sensitivity, specificity and predictive value of laparoscopy for the detection of liver metastases. Cancer 1986;58:1-6.

35. van Dijkum EJ, de Wit LT, van Delden OM, Rauws EA, van Lanschot JJ, Obertop H, et al. The efficacy of laparoscopic staging in patients with upper gastrointestinal tumours. Cancer 1997;79: 1315-9.

36. Feussner H, Omote K, Fink U, Walker SJ, Siewert JR. Pretherapeutic laparoscopic staging in advanced gastric carcinoma. Endoscopy 1999;31:389-91.

37. Lehnert T, Rudek B, Kienle P, Buhl K, Herfarth C. Impact of diagnostic laparoscopy on the management of gastric cancer: prospective study of 120 consecutive patients with primary gastric adenocarcinoma. Br J Surg 2002;89:471-5.

38. Hulscher JB, Nieveen van Dijkum EJ, de Wit LT, van Delden $\mathrm{OM}$, van Lanschot JJ, Obertop $\mathrm{H}$, et al. Laparoscopy and laparoscopic ultrasonography in staging carcinoma of the gastric cardia. Eur J Surg 2000;166:862-5.

39. Finch MD, John TG, Garden OJ, Allan PL, Paterson- Brown S. Laparoscopic ultasonography for staging gastroesophageal cancer. Surgery 1997;121:10-7.

40. Lavonius MI, Gullichsen R, Salo S, Sonninen P, Ovaska J. Staging of gastric cancer: a study with spiral computed tomography, ultrasonography, laparoscopy and laparoscopic ultrasonography. Surg Laparosc Endosc Percutan Tech 2002;12:77-81. 